\title{
AUTOMATIC CORRECTION OF IMAGE INTENSITY NON-UNIFORMITY BY THE SIMPLEST TOTAL VARIATION MODEL*
}

\author{
ANA BELÉN PETRO ${ }^{\dagger}$, CATALINA SBERT $^{\dagger}$, AND JEAN-MICHEL MOREL ${ }^{\ddagger}$
}

\begin{abstract}
Extending if possible the spectacular applications of the total variation model in image processing, this paper proposes an extension of the TV model to attenuate the effects of non-uniform illumination in digital images. We compare the simplest possible linear algorithm and the simplest possible total-variation based algorithm. The comparison demonstrates once again that the total variation model improves images with minimal halo artifacts. We show that with a single contrast parameter (which keeps the same value in most experiments), the total variation model delivers state of the art results. They compare favourably to results obtained with more complex algorithms. Our algorithm is designed for all kinds of images, but with the special specification of making minimal image detail alteration thanks to a first order fidelity term, instead of the usual zero order term. Experiments on non-uniform medical images and on hazy images images illustrate significant perception gain.
\end{abstract}

Key words. Total variation, Poisson equation, gradient-based methods, non-uniform illumination, color perception theory.

AMS subject classifications. 35J, 42, 49.

1. Introduction. The gradient-based image processing techniques and the Poisson equation have been used in several areas of image processing. The motivation to use gradients is based on the Retinex theory of Land and McCann [18] which states that the human visual system is more sensitive to illumination differences than to absolute luminance. A number of applications based on this technique have been developed, such as high dynamic range compression [9], Poisson image editing [25], Retinex [23], [19], contrast enhancement [24], [3], shadow removal [10], cartoon-texture decomposition [31], etc.

In the gradient domain, the goal is to find the function $u$ whose gradient field is the nearest to some given vector field $\mathbf{V}$ in $L_{p}$ norm. This problem is solved by minimizing the functional

$$
\int_{\Omega}\|\nabla u-\mathbf{V}\|^{p} d x=\int_{\Omega}\left(\sum_{i}\left|(\nabla u)_{i}(x)-V_{i}(x)\right|^{2}\right)^{p / 2} d x
$$

and for the special case $p=2$, the solution satisfies a Poisson equation. Depending on the choice of the vector field $\mathbf{V}$ many applications have been developed, like for example tuning large gradients for high dynamic range compression, amplifying small gradients for increasing the contrast, seamless copy-paste or photomontage copying the gradient of some part of an image into another image.

Illumination is one of the most important factors affecting the appearance of an image. Adjusting the illumination conditions to obtain a uniform contrast across the entire image can be difficult or impossible, depending on the imaging system. If illumination is not uniform in the spatial domain, similar objects at different locations

\footnotetext{
* Received February 3, 2013; accepted for publication October 16, 2013.

${ }^{\dagger}$ Department of Mathematics and Computer Sciene, Universitat de les Illes Balears, Palma de Mallorca, Spain (\{anabelen.petro; catalina.sbert\}@uib.es).

${ }_{\ddagger}$ Centre de Mathématiques et leur applications (CMLA), ENS Cachan, Paris, France (morel@ cmla.ens-cachan.fr).
} 
in the acquired image take on different intensity values, making it difficult to recognize the objects based on the intensity values. This kind of artifact may be present in magnetic resonance (MR) images due to the inhomogeneity of the applied magnetic field, in microscopy images due to the nonuniform illumination of the light source, or in underwater photography since the light absorption properties of the water varies as a function of the distance from the camera.

Medical image analysis methods, such as segmentation, are highly sensitive to this kind of inhomogeneities. This is why a large number of methods for intensity inhomogeneity correction have been proposed in the past twenty years. We refer to a recent review [30] of such methods applied to MRI images.

In this work, we propose and investigate a very simple gradient domain method that tries to eliminate the effect of nonuniform illumination and at the same time preserves image detail. The goal of the retinex-like methods is relatable to the cartoontexture decomposition method. Nevertheless, the retinex method does not aim at an additive decomposition. The nonuniform illumination present in the image does not correspond to a background image, or to a cartoon version of the image. Our proposed nonuniform illumination elimination result is a unique image where the image details (cartoon and texture as well) are preserved and enhanced. In the Zhang et al. [32] cartoon+texture method, the underlying model is an additive decomposition method by the so called ROF $T V+L^{2}$ model. Similarly, the celebrated Yin et al. [31] decomposition method develops a $T V+L^{1}$ model. It is easy to see that our model here is different by comparing the form of the functionals:

- $T V+L^{2}: \min _{u, v \mid f=u+v} \int\|\nabla u\|+(f-u)^{2}$;

- $T V+L^{1}: \min _{u, v \mid f=u+v} \int\|\nabla u\|+|f-u|$;

- our model, $p=1,2: \min _{u} \int \| \nabla(u-f)||^{p}+|u-\bar{u}|^{2}, p=1$ or 2 , where $\bar{u}$ is the mean of $u$.

The main difference of the proposed retinex model with the cartoon + texture models will be that the solution $u$ is constrained to have its gradient as close as possible to the data $f$. Instead, in the $T V-L^{p}$ models the emphasis is on limiting the total variation of $u$, the "cartoon", while much of the textural gradient of $f$ is transferred to $v$. So the gradient of the original data $f$ is split between cartoon and texture. In short in a cartoon+texture model the solution is given by two functions $u$ and $v$, while in our model there is only one solution function, $u$, which is required to be as similar to $f$ as possible. Considering for example the experiment in figure 1 , it is clearly seen that the nonuniform illumination, eliminated by our proposed method, does not correspond to a background image or to a cartoon image. Indeed, the main result of the method stills contains the cartoon and the texture part together. The difference of original and restored contains the non uniform illumination and only a minor part of the gradient information.

We now give a detailed justification of the proposed model. Preserving the details is more or less equivalent to preserving the image gradient. Thus the functional should have a term of the type (1), with $\mathbf{V}=\nabla f$. To make the image illumination more uniform, the simplest way that comes to mind is to minimize the image variance

$$
\frac{1}{|\Omega|} \int_{\Omega}(u-\bar{u})^{2} d x
$$

where $\bar{u}$ represents the mean value of the image and where the domain of integration 

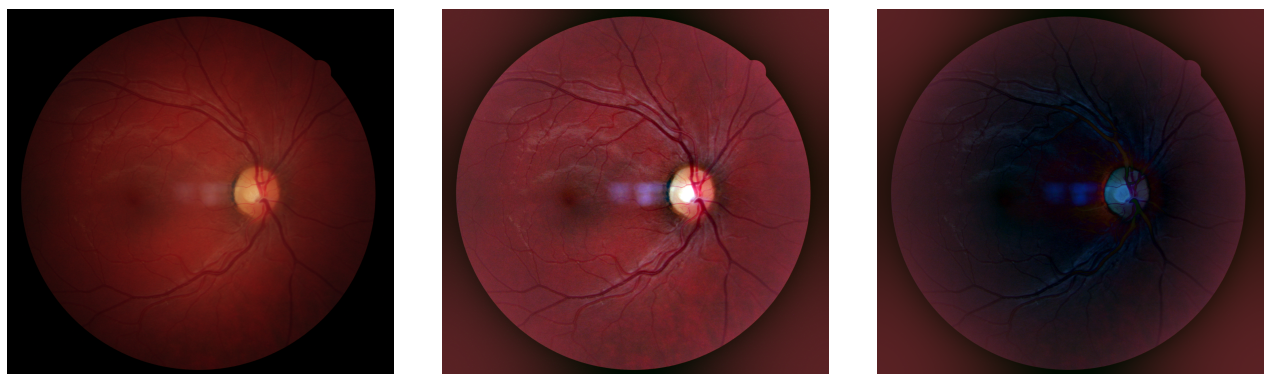

FIG. 1. Left: Original image. Center: Result of our method. Right: Difference between previous images. This difference keeps as little as possible information from the original image gradient. The cartoon part and the texture part are still present together in the corrected image.

$\Omega$ is the image domain. This leads to combine the variational terms (1-2) into a single functional

$$
J(u)=\int_{\Omega}\|\nabla u-\nabla f\|^{p} d x+\frac{\lambda}{2} \int_{\Omega}(u-\bar{u})^{2} d x,
$$

where $\lambda$ is a constant that controls the trade-off between both terms. In this work, we shall consider and compare the linear case $p=2$, and the nonlinear case $p=1$. For $p=2$, when minimizing the first term of the above functional alone, the solution is $u=f+k$ where $k$ is an arbitrary constant, but if we stretch the solution range to the interval $[0,255]$ the result is equivalent to stretching the original image $f$ to this interval. Minimizing only the variance term of the functional (3) yields instead a constant image. By minimizing both terms together, it is expected that image detail will be maintained, but that the image variance will decrease. As a consequence, the solution of (3) will generally satisfy $\max u-\min u<\max f-\min f$. It follows that in practice one can stretch linearly the dynamics of the image $u$ minimizing (3), by applying an affine function $v=a u+b$ to the result so that $\min v=0$ and $\max v=255$. In that way, we shall observe in all examples that $a>1$, so that a serious enhancement of the details of $f$ is obtained. We shall therefore call solution of (3) the image $v$ obtained from $u$ minimizing (3).

We will show that this model acts as a high pass filter. In the literature one can find similar Wiener-like filters, [24], [22], [9], [23]. Other models like the so called multiscale retinex methods [14] are used to remove the low frequency illumination components while preserving the high frequency reflectance components. They can also be seen as nonlinear high pass filters, and the same consideration applies to filters that try to model the human visual system [27], [12].

The paper is organized as follows Section 2 presents the variational model and solves exactly in the Fourier domain, for $p=2$, its associated screened Poisson equation. Section 3 presents the model for $p=1$ and solves it by Chambolle's projection algorithm. Section 4 presents experimental comparative results and a final discussion.

2. The model for $p=2$. Given an image $f$, the goal is to find a function $u$ whose vector gradient is close to the vector gradient of $f$, but with reduced variance to compensate for illumination inhomogeneities. The objective function to minimize is

$$
J(u)=\int_{\Omega}\|\nabla u-\nabla f\|^{2} d x+\frac{\lambda}{2} \int_{\Omega}(u-\bar{u})^{2} d x .
$$


The Euler-Lagrange equation is in appearance nonlinear but we can simplify w.l.o.g. the model by considering that the mean value of $u$ coincides with the mean value of $f$. With this condition, the function $u$ that minimizes the functional $J$ satisfies the Euler-Lagrange equation

$$
\lambda(u-\bar{f})-\Delta u+\Delta f=0, \quad \text { over } \quad \Omega
$$

with homogeneous Neumann boundary condition

$$
\frac{\partial u}{\partial \mathbf{n}}=0, \quad \text { over } \quad \partial \Omega
$$

where $\mathbf{n}$ is the normal vector to the boundary. The equation (5) is known as a screened Poisson equation.

2.1. Independence of the mean value. It remains to prove that imposing a mean value to the solution is irrelevant. Consider the problem

$$
\begin{cases}\lambda u-\Delta u=-\Delta f+\lambda K & \text { in } \Omega \\ \frac{\partial u}{\partial \mathbf{n}}=0 & \text { in } \partial \Omega\end{cases}
$$

where $K$ is a constant and $\lambda>0$. It is well known that the problem (6) has a unique solution when $\lambda>0$. Let $u_{1}$ and $u_{2}$ be two solutions of (6) with constants $K_{1}$ and $K_{2}$ respectively. Then it is easily checked that

$$
u_{1}-u_{2}=K_{1}-K_{2} .
$$

Working (e.g.) with eight-bit digital images, the range of values of the image is the interval $[0,255]$. When solving the problem (6) in the image context, the final solution must be stretched on this range as mentioned above, to get the best out of relative the contrast gain attained by the method. Consider $u_{1}$ and $u_{2}$, solutions of (6) for two constants $K_{1}$ and $K_{2}$ respectively and let $\overline{u_{1}}, \overline{u_{2}}$ be the associated solutions in the range $[0,255]$. Setting $M_{i}=\max u_{i}$ and $m_{i}=\min u_{i}, i=1,2$ we have

$$
\overline{u_{i}}=\frac{255}{M_{i}-m_{i}}\left(u_{i}-m_{i}\right), \quad i=1,2,
$$

but $M_{1}=M_{2}+K_{1}-K_{2}$ and $m_{1}=m_{2}+K_{1}-K_{2}$, which implies $\overline{u_{1}}=\overline{u_{2}}$. We conclude that the final solution is independent of the constant $K$, so from now on we will consider $K=0$.

2.2. The screened Poisson equation as a high pass filter. This section analyzes the solution of the screened Poisson equation

$$
\lambda u-\Delta u=-\Delta f
$$

as the result a high pass filter of the function $f$, and more precisely as a "centersurround" filter, namely the subtraction from the image of the same image convolved with a local radial low pass filter. Solving the equation in the Fourier domain yields

$$
\widehat{u}=\frac{w_{x}^{2}+w_{y}^{2}}{\lambda+w_{x}^{2}+w_{y}^{2}} \widehat{f}
$$




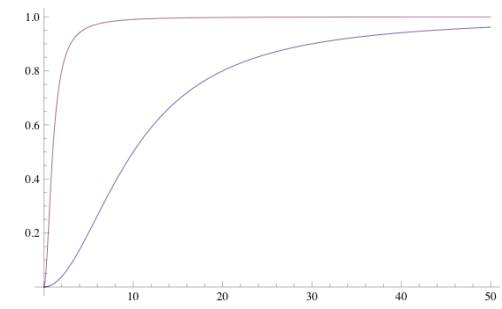

FIG. 2. The frequency domain filter for $\lambda=0.1$ and $\lambda=50$.

The frequency filter acts as a high pass filter when $\lambda$ increases as illustrated in Figure 2. Starting from equation (7) the frequency filter can be decomposed as

$$
\frac{w_{x}^{2}+w_{y}^{2}}{\lambda+w_{x}^{2}+w_{y}^{2}}=1-\frac{\lambda}{\lambda+w_{x}^{2}+w_{y}^{2}} .
$$

Computing the inverse Fourier transform of this filter indicates that the solution of the screened Poisson equation is obtained by convolving the image $f$ with a spatial domain filter. More precisely, the inverse Fourier transform of the first term (8) is a Dirac delta function $\delta(x, y)$. The second term is radially symmetric and interpretable using the Hankel transform [4]. Denoting by $\mathcal{H}\{f\}$ the Hankel transform of $f(r)$, we have

$$
\mathcal{H}^{-1}\left\{\frac{1}{a^{2}+r^{2}}\right\}=K_{0}(\text { ar })
$$

where $K_{0}$ is the zeroth order modified Bessel function of second kind. Then the inverse Fourier transform of the frequency filter (8) is

$$
\delta(x, y)-\frac{1}{2 \pi} K_{0}\left(\sqrt{\lambda\left(x^{2}+y^{2}\right)}\right) .
$$

Convolving the filter (9) with $f$ yields the solution of the screened Poisson equation

$$
u(x, y)=f(x, y)-\frac{1}{2 \pi} K_{0}\left(\sqrt{\lambda\left(x^{2}+y^{2}\right)}\right) * f(x, y) .
$$

Thus, the solution is the difference between the data function and a blurred version of it with the $K_{0}$ filter. This filter acts as a center-surround filter, as illustrated in Figure 3. Center-surround filters of this kind model the human perception of colours [15], [3].

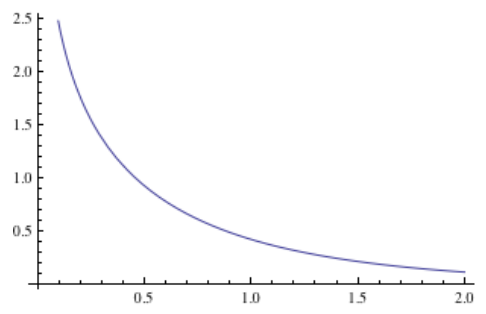

FiG. 3. The zeroth order modified Bessel function of second kind $K_{0}(r)$ 
2.3. Numerical solution by DFT. We want to solve the problem

$$
\begin{cases}\lambda u-\Delta u=-\Delta f & \text { in } \Omega \\ \frac{\partial u}{\partial \mathbf{n}}=0 & \text { in } \partial \Omega\end{cases}
$$

where $\lambda>0$. We can solve the screened Poisson equation (11) using the discrete Fourier transform. Like in [24], the Neumann boundary condition is implicitly imposed by extending the original image symmetrically across its sides, so that the extended image, which is four times bigger, becomes symmetric and periodic. The discrete Fourier transform (DFT) permits to compute directly the Fourier coefficients of a band-limited and $(J, L)$-periodic function $u$ from its samples $u_{j l}$ on a $J \times L$ grid. The output of the algorithm is an image which is the result of applying the screened Poisson equation to each color channel separately, followed by a simplest color balance [20] with a $0.2 \%$ of saturation. The discrete Fourier transform (DFT) permits to compute directly the Fourier coefficients of a band limited and $(J, L)$-periodic function $u$ from its samples $u_{j l}$ on a $J \times L$ grid. The solution in the Fourier domain is given by

$$
\widehat{u}_{m n}=\frac{\left(\left(\frac{2 \pi m}{J}\right)^{2}+\left(\frac{2 \pi n}{L}\right)^{2}\right)}{\left(\lambda+\left(\frac{2 \pi m}{J}\right)^{2}+\left(\frac{2 \pi n}{L}\right)^{2}\right)} \widehat{f}_{m n} .
$$

Applying the discrete inverse Fourier transform yields the solution $u$.

In eight bit digital images the range is $[0,255]$. The best results are obtained when a certain small percentage of pixels are saturated at 255 and 0 , as shown in [20]. The proposed model is applied to the result of a simplest color balance with $0.2 \%$ saturation, and the final result is obtained by applying to the solution a simplest color balance again.

3. The model for $p=1$. For $p=1$, the objective function to minimize is

$$
J(u)=\int_{\Omega}\|\nabla u-\nabla f\| d x+\frac{\lambda}{2} \int_{\Omega}(u-\bar{f})^{2} d x,
$$

where $\lambda$ is a constant that controls the trade-off between both terms. Setting $v=u-f$ permits to rewrite the functional (13) in the classic "TV-denoising" [28] form as

$$
J(v)=\int_{\Omega}\|\nabla v\| d x+\frac{\lambda}{2} \int_{\Omega}(v-(\bar{f}-f))^{2} d x,
$$

for which numerous numerical approaches have been proposed. Classical techniques use the associated Euler-Lagrange equation. Rudin, Osher and Fatemi [28] used a time marching scheme to reach the steady state of the parabolic equation. This method can be slow. Chan, Golub and Mulet [7] proposed to introduce an additional variable to linearize the term div $\left(\frac{\nabla v}{|\nabla v|}\right)$. This method can be viewed as a dual method. In an extended dual formulation Chambolle [5] proposed a now-classic fast algorithm which we shall use here for the sake of simplicity. More recently Goldstein and Osher [13] have shown that the Bregman iteration can be used to solve constrained minimization problems; they propose a "Split Bregman" method and have applied this technique to the Rudin-Osher-Fatemi functional for image denoising. The Split-Bregman method can be tested on any uploaded image with the demo associated with the paper [11], which gives all implementation details. 
3.1. Solution by Chambolle's projection algorithm. For our application we used the dual approach introduced by Chambolle in [5]. In the discrete setting, denote by $X=\mathbb{R}^{N \times N}$, so that the image $u$ belongs to $X$ and the vector field $p$ to $Y=X \times X$. The discrete total variation is

$$
F_{d}(u)=\sum_{1 \leq i, j \leq N}\left|(\nabla u)_{i j}\right|=\max _{p \in Y,\left|p_{i j}\right| \leq 1, \forall(i, j)}<p, \nabla u>_{Y} .
$$

We introduce the discrete divergence div as the dual operator of $\nabla$, i.e., for every $p \in Y$ and $u \in X$ we have

$$
<-\operatorname{div} p, u>_{X}=<p, \nabla u>_{Y}
$$

Then, we have

$$
F_{d}(u)=\max _{p \in V}<\operatorname{div} p, u>
$$

where

$$
V=\left\{p \in Y,\left|p_{i j}\right|^{2}-1 \leq 0, \forall(i, j)\right\} .
$$

Chambolle uses a dual formulation for solving the problem

$$
\min _{u \in X} F_{d}(u)+\frac{\lambda}{2}\|u-g\|_{X}^{2}
$$

where $g \in X$. He shows [5] that the solution $u$ of (16) is given by

$$
u=g-\Pi_{\frac{1}{\lambda} K}(g)
$$

where $\Pi_{\frac{1}{\lambda} K}(\cdot)$ is the orthogonal projection onto the convex set $\frac{1}{\lambda} K$, with

$$
K=\left\{\operatorname{div} p: p \in Y,\left|p_{i, j}\right| \leq 1, \forall i, j\right\} .
$$

To compute this projection a semi-implicit fixed point iteration is applied: $p^{0}=0$ and

$$
p_{i, j}^{n+1}=\frac{p_{i, j}^{n}+\nu \nabla\left[\operatorname{div} p^{n}-\lambda g\right]_{i, j}}{1+\nu\left|\nabla\left[\operatorname{div} p^{n}-\lambda g\right]_{i, j}\right|} .
$$

It is shown in [5] that if $\nu<1 / 8$, then $g-\operatorname{div} p^{n} / \lambda$ converges to the solution of (16). In practice, convergence is generally observed as long as $\nu<1 / 4$. In a subsequent paper Chambolle [6] has proposed a modification of his projection algorithm, and Aujol [1] shows that this modification can be seen as a particular case of a more general algorithm proposed 30 years ago by Bermudez and Moreno [2]. To solve (14) this algorithm was used with $g=\bar{f}-f, \nu=0.24$, and the stopping criterion

$$
\left\|p^{n+1}-p^{n}\right\| \leq 10^{-3}\left\|p^{1}-p^{0}\right\| .
$$

The obtained solution by the minimization is $v=\bar{f}-f-\operatorname{div} p^{n} / \lambda$. Finally, we get back the solution of (13) by $u=v+f$.

4. Results. In this section we present some applications of the models, and a comparison of them. These include non uniform illumination correction, contrast enhancement and edge detection. 

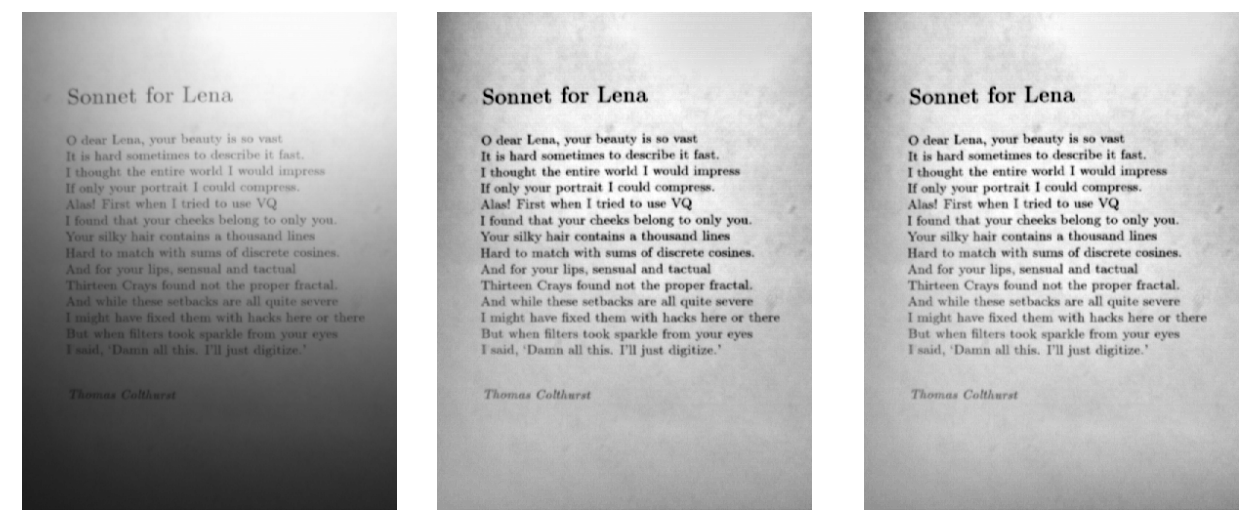

FIG. 4. Left: Original Image. Middle: Result with $p=2, \lambda=0.0005$. Right: Result with $p=1$, $\lambda=0.001$

4.1. Non uniform illumination correction. Illumination is one of the most important factors affecting the appearance of an image. Figure 4 shows an example of a text image with nonuniform illumination. The result of the two models $p=2$ and $p=1$ are similar. In Figure 4 we show the result for $p=2$ with $\lambda=0.0005$ and for $p=1$ with $\lambda=0.001$ because both results have the same variance.

In ophthalmology the use of advanced fundus cameras has become a standard imaging modality to diagnose diseases such as glaucoma, diabetic retinopathy etc. A good diagnosis depends on the quality of the image, which requires a good contrast of the relevant objects on the retina such as the blood vessels or the optic disk, and is favoured by a uniformity of the retina illumination. The classic methods to process this kind of images mostly try to estimate the illumination or the bias field, using the commonly used terminology, and subtract it from the original image to obtain the final result [17], [16]. There are several works whose aim is the detection of retinal blood vessels, for example the work of Zhang et. al. [32], which results might be improved using an initial image with non uniform illumination correction.

Figure 5 shows an example of a retina image and the result after applying our model. We compare the results with the above mentioned classic method [16]. Clearly the blood vessels are more visible in dark regions and the result can be used as a preprocessing step in the blood vessel segmentation. The image and the result of the method in [16] come from the public fundus images database DRIIL (Digital Retinal Images for ILlumination correction http://projects.ubmi.feec.vutbr.cz/ ophthalmo).

Another example for potential applications is given by magnetic resonance images (MRI), where the intensity inhomogeneity is due for example to radio frequency inhomogeneities or to variations in the static magnetic field. Many works have been dedicated to the correction of this kind of inhomogeneity. Vovk et al. [30] propose a classification of the correction methods into prospective methods, that attempt to improve the image acquisition process, and the retrospective methods that work with the information of the acquired image. The retrospective methods can be classified into filtering, surface fitting, segmentation and histogram based. The filtering methods assume that the nonuniform illumination is a low frequency artifact that must be removed. This is achieved by subtracting from the image its low-pass filtered version. To this group belong the homomorphic filtering and the unsharp masking. The 

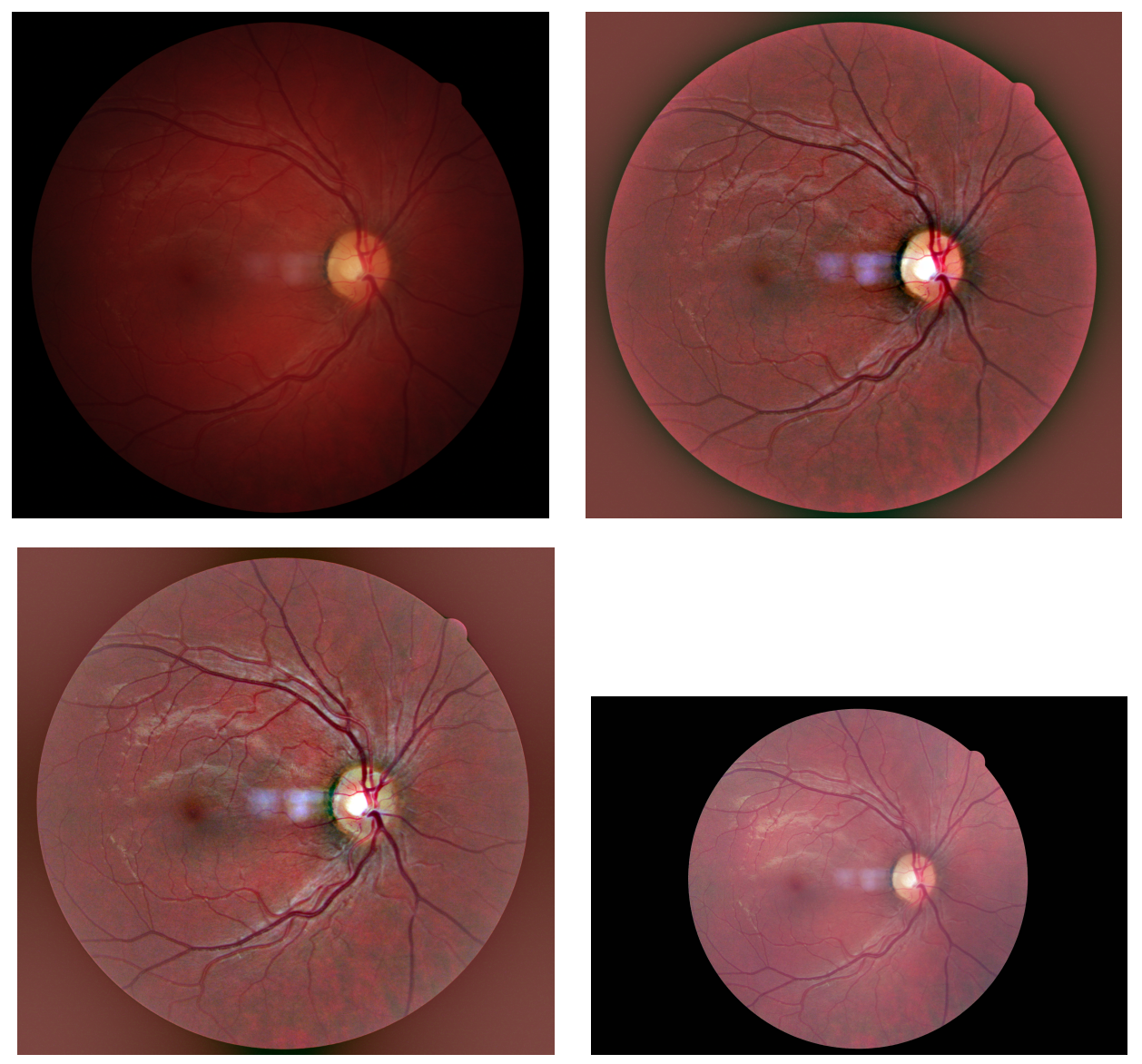

FIG. 5. Top left: original image. Top right: result for $p=2$ with $\lambda=0.0005$. Bottom left: result for $p=1$ with $\lambda=0.001$. Notice that with $p=1$ the halo effect near the object's boundary is much reduced. Bottom right: result of the model in [16].

method we have proposed for $p=2$ belongs to this group, as explained in Section 2.2. Note that when $\lambda$ increases, the support of this filter becomes smaller.

Figure 6 shows the result of our models on MRI images with intensity inhomogeneities. Clearly the dark regions become more visible and image details are preserved.
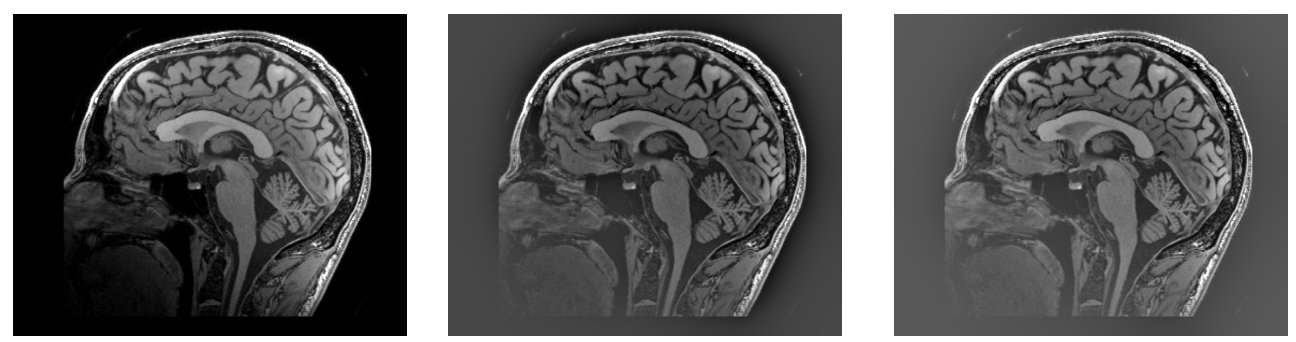

FIG. 6. Left: original image. Middle: Result for $p=2$ with $\lambda=0.001$. Right: Result for $p=1$ with $\lambda=0.001$. Again, notice the absence of halo effect with $p=1$. 
4.2. Contrast enhancement results. The recently proposed contrast enhancement methods give increasingly good results [20], [24], [21], [14], [27], etc. In this section we compare the result of the linear model, $p=2$, with $p=1$, with the state of the art automatic color enhancement (ACE) [27] and with the equally classic multiscale retinex with color restoration (MSRCR) [14]. ACE results were obtained from the online fast implementation of this algorithm [12] and we have implemented our own (MSRCR).

Figure 7 presents a low contrast image and the corresponding results of ACE, MSRCR and our model with the parameter $\lambda=0.0001$. Observe that the high frequencies (for example in the letters on the fire-fighter helmet) are better preserved with our model. Another example of contrast enhancement is shown in Figure 8.
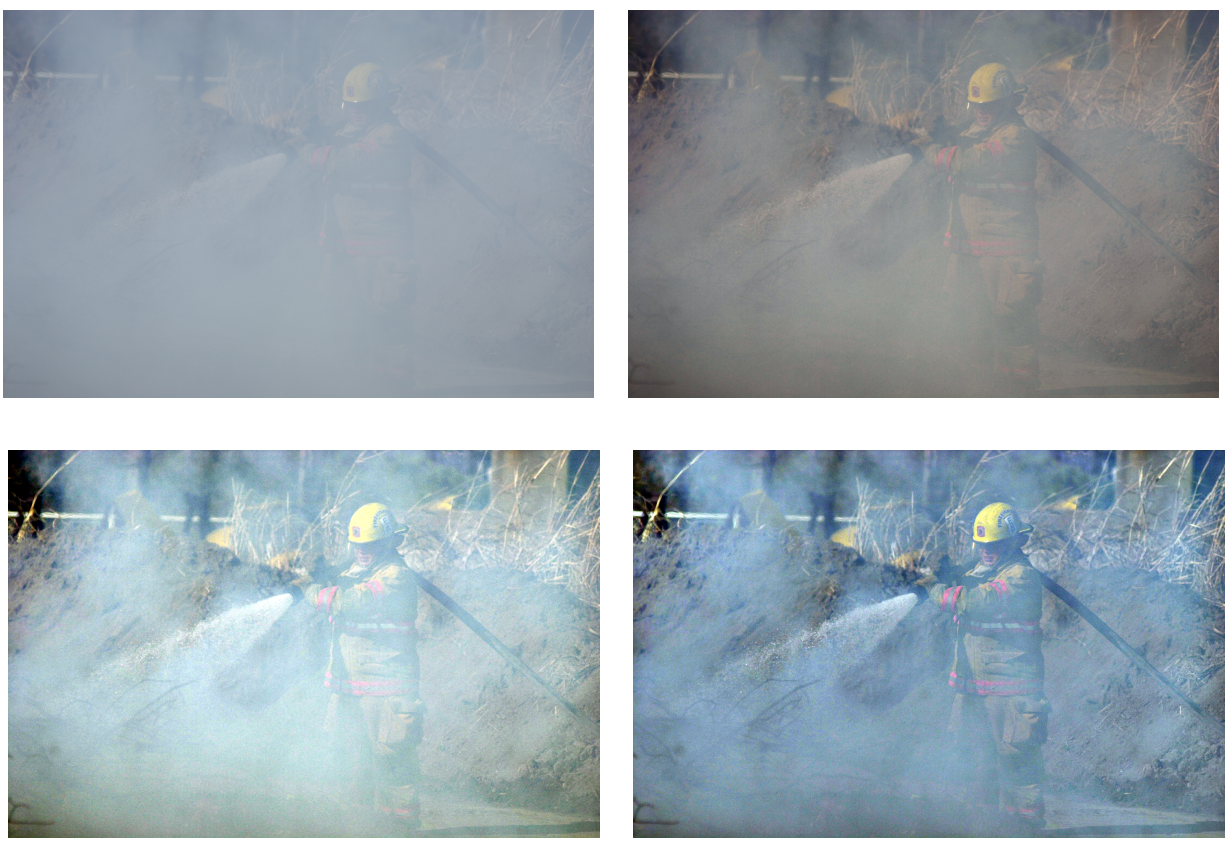

FIG. 7. Top left: Original Image. Top right: Result of ACE. Bottom left: Result with MSRCR (multiscale retinex). Bottom right: our model with $\lambda=0.0001$.

Observe that the best result in this case is with the ACE method as the MSR method leaves a very saturated image.

4.3. Comparison of the models and conclusions. In unsharp masking [26] the original image is enhanced by subtracting a smooth version of the original image, which always also gives a center-surround filter interpretation. As we have shown in a previous section our linear model acts as an unsharp mask when $\lambda$ increases. The unsharp models can produce artifacts, such as halos around the edges. These artifacts are nevertheless reduced with our total variation model $(p=1)$, since it keeps the sharp edges. Indeed the TV regularization term penalizes all intensity variations in a uniform way, in contrast to the linear model which penalizes larger changes more heavily. A model that does not preserve edges well enough will inevitably cause halo artifacts. Figure 9 shows an example of this halo artifact for the linear model. It also shows that this effect does not occur with the TV model.

Figure 10 shows an extract of the image in Figure 7, and we can observe again 

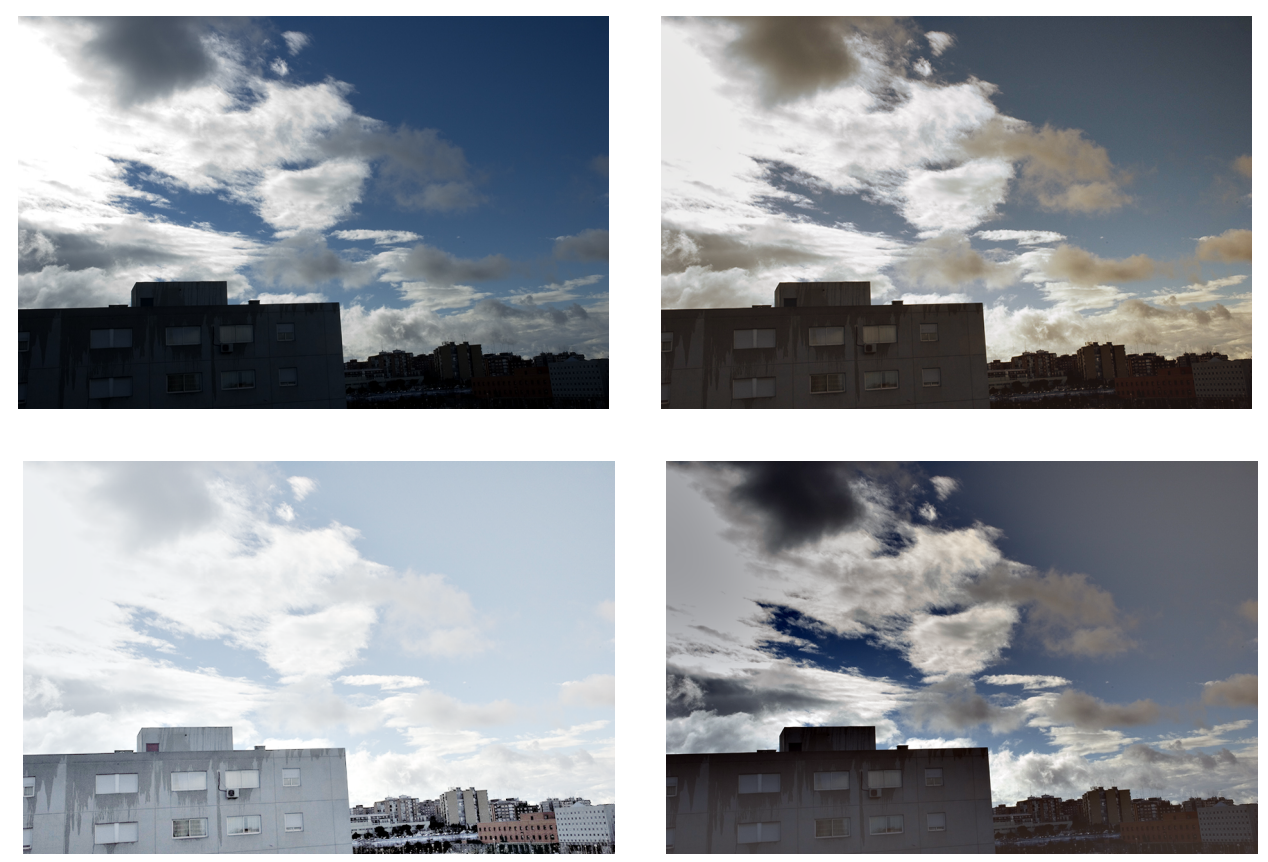

Fig. 8. Top left: Original Image. Top right: Result of ACE. Bottom left: Result with MSRCR. Bottom right: our model with $\lambda=0.0001$. The retinex result is the most readable, but looses perhaps too much contrast in the sky, removing also completely the twilight effect.
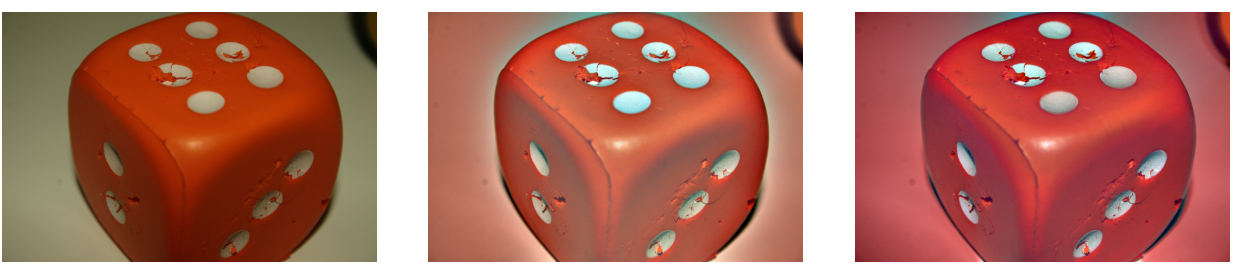

FIG. 9. Left: original image. Middle : result for the linear model with $\lambda=0.001$. Right : result for the TV model with $\lambda=0.001$. The effects of the linear and total variation model are good and very similar, but with again a reduced halo effect for $p=1$.

the halo effect on the fire-fighter's helmet for the linear model. Thus, the experiments confirm once again the observations made in [22] as to the superiority of the $L^{1}$ model to deal with image contrast without introducing artifacts, and add one new item to the long list of remarkable applications [8] of the total variation to image processing.

Acknowledgment. The authors were supported by the Ministerio de Ciencia e Innovación under Grant TIN2011-27539, by MISS project of Centre National d'Etudes Spatiales, the Office of Naval Research under Grant N00014-97-1-0839 and by the European Research Council, advanced grant "Twelve labours".

\section{REFERENCES}

[1] J. F. Aujol, Some first-order algorithms for total variation based image restoration, Journal of Mathematical Imaging and Vision, 34 (2009), pp. 307-327. 

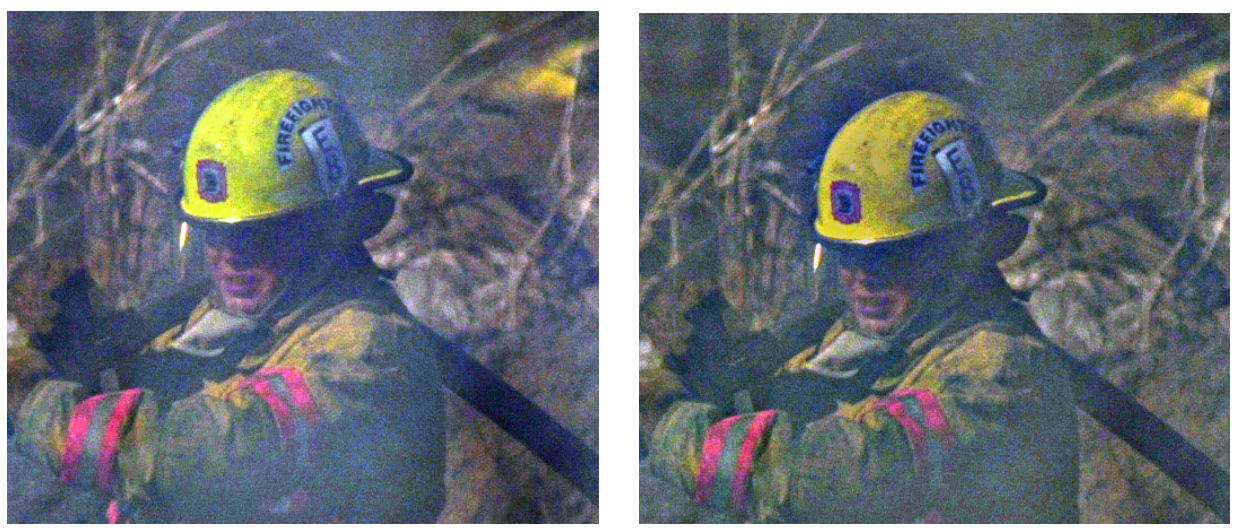

FIG. 10. Left: Result with the TV model. Right: Result with the linear model. Notice the color halo above the helmet with the linear model.

[2] A. Bermúdez and C. Moreno, Duality methods for solving variational inequalities, Computers \& Mathematics with Applications, 7 (1981), pp. 43-58.

[3] P. Bhat, B. Curless, M. Cohen, and C. Lawrence, Fourier analysis of the $2 D$ screened Poisson equation for gradient domain problems, Proceedings of ECCV'08, Part II, pp. 114$128,2008$.

[4] R. Bracewell, The Fourier Transform and Its Applications, second edition, Mcgraw-Hill, 1986.

[5] A. Chambolle, An algorithm for total variation minimization and applications, J. of Mathematical Imaging and Vision, 20 (2004), pp. 89-97.

[6] A. Chambolle, Total variation minimization and a class of binary MRF models, In EMMCVPR'05, Lecture Notes in Computer Science, 3757 (2005), pp. 136-152.

[7] T. Chan, G. Golub, And P. Mulet, A nonlinear primal-dual method for total variation based image restoration, SIAM Journal of Computing 20 (1999), pp. 1964-1977.

[8] T. Chan, S. Esedoglu, F. Park, And A. Yip, Recent developments in total variation image restoration, Mathematical Models of Computer Vision 17, Springer Verlag, 2005.

[9] R. Fattal, D. Lischinski, and M. Werman, Gradient Domain High Dynamic Range Compression, ACM Transactions on Graphics 2002, 21, 3, pp. 249-256.

[10] G. Finlayson, S. Hordley, And M. Drew, Removing shadows from image, Proc. of ECCV 2002, pp. 823-836, 2002.

[11] P. Getreuer, Rudin-Osher-Fatemi total variation denoising using split Bregman, Image Processing On Line 2012. http://dx.doi.org/10.5201/ipol.2012.g-tvd

[12] P. Getreuer, Automatic Color Enhancement (ACE) and its Fast Implementation, Image Processing On Line, 2012. http://dx.doi.org/10.5201/ipol.2012.g-ace

[13] T. Goldstein And S. OsheR, The Split Bregman method for $L^{1}$-regularized problems, SIAM Journal of Imaging Sciences, 2 (2009), pp. 323-343.

[14] D. J. Jobson, Z. Rahman, And G. A. Woodell, A multiscale retinex for bridging the gap between color images and the human observation of scenes, IEEE Transactions on Image Processing , 6 (1997), pp. 965-976.

[15] D. J. Jobson, Z. Rahman, And G. A. Woodell, Properties and performance of a center/surround retinex, IEEE Transactions on Image Processing, 6 (1997), pp. 451-462.

[16] R. Kolar, J. Odstrcilik, J. Jan, and V. Harabis, Illumination Correction and Contrast Equalization in Color Fundus Images, Proc. of the European Signal Processing Conference, pp. 298-302, 2011.

[17] L. KuBecka, J. Jan, AND R. KolaR, Retrospective illumination correction of retinal images, International Journal of Biomedical Imaging, 9 (2010), 10 pages.

[18] E. H. LAND, The Retinex Theory of Color Vision, Scientific American, 236 (1977), pp. 108-128.

[19] N. Limare, J. M. Morel, A.-B. Petro, And C. Sbert, Retinex Poisson equation: a model for color perception, Image Processing On Line, 2011. http://dx.doi.org/10.5201/ipol. 2011.1mps_rpe

[20] N. Limare, J. L. Lisani, J. M. Morel, A.-B. Petro, and C. Sbert, Simplest color balance, Image Processing On Line, 2011. http://dx.doi.org/10.5201/ipol.2011.1lmps-scb 
[21] J. L. Lisani, A.-B. Petro, and C. Sbert, Color and contrast enhancement by controlled piecewise affine histogram equalization, Image Processing On Line, 2012.

[22] W. Ma, J. M. Morel, S. Osher, and A. Chien, An $L^{1}$-based variational model for Retinex theory and its application to medical images, IEEE Conference on Computer Vision and Pattern Recognition, pp. 153-160, 2011.

[23] J. M. Morel, A.-B. Petro, And C. Sbert, A PDE formalization of the retinex theory, IEEE Trans. on Image Processing, 19 (2010), pp. 2825-2837.

[24] J. M. Morel, A.-B. Petro, and C. Sbert, Fourier implementation of Poisson image editing, Pattern Recognition Letters, 33 (2012), pp. 342-348.

[25] P. Pérez, M. Gangnet, and A. Blake, Poisson image editing, ACM Transactions on Graphics, 22 (2003), pp. 313-318.

[26] A. Polesel, G. Ramponi, and V. J. Mathews, Image enhancement via adaptive unsharp masking, IEEE Transactions on Image Processing, 9 (2000), pp. 505-510.

[27] A. Rizzi, C. Gatta, And D. Marini, A new algorithm for unsupervised global and local color correction, Pattern Recognition Letters, 24 (2003), pp. 1663-1677.

[28] L. Rudin, S. Osher, AND E. FATEMI, Nonlinear total variation based noise removal algorithms, Physica D, 60 (1992), pp. 259-268.

[29] J. G. Sled, A. P. Zijdenbos, And A. C .Evans, A nonparametric method for automatic correction of intensity nonuniformity in MRI data, IEEE Transactions on Medical Imaging, 17 (1998), pp. 87-97.

[30] U. Vovk, F. Pernus, B. Likar, A review of methods for correction of intensity inhomogeneity in MRI, IEEE Transactions on Medical Imaging, 26 (2007), pp. 405-421.

[31] W. Yin, D. Goldfarb, and S. Osher, Total variation based image cartoon-texture decomposition, DTIC Document, 2005.

[32] Y. Zhang, W. Hsu, And M. L. Lee, Detection of retinal blood vessels based on nonlinear projections, Journal of Signal Processing Systems, 55 (2009), pp. 103-112. 
A. B. PETRO, C. SBERT, AND J.-M. MOREL 